\title{
Management of Fungal Keratitis in Pediatric Patients
}

\author{
Parisa Badiee, ${ }^{1}$ Mahmood Nejabat, ${ }^{2}$ and Maral Choopanizadeh ${ }^{1, *}$ \\ ${ }^{1}$ Professor Alborzi Clinical Microbiology Research Center, Shiraz University of Medical Sciences, Shiraz, Iran \\ ${ }^{2}$ Ophthalmology Department, Khalili Hospital, Shiraz University of Medical Sciences, Shiraz, Iran \\ Corresponding author: Maral Choopanizadeh, Prof, Alborzi Clinical Microbiology Research Center, Namazee Hospital Zand Ave, Shiraz, Iran. Tel: +98-7136474292, Fax: \\ +98-7136474303, E-mail: choopanizadeh@sums.ac.ir
}

Received 2016 December 10; Revised 2017 February 14; Accepted 2017 February 27.

\begin{abstract}
Context: Fungal keratitis is an uncommon infection with high morbidity in pediatric patients. Trauma is the most common risk factor for infection in children. Thus, rapid diagnosis should be made by scoring clinical features and mycological examination. This review discusses the management of fungal keratitis in pediatric patients.

Evidence Acquisition: In this narrative review, the most recently published data on pediatric fungal keratitis, respective prominent signs and symptoms, etiologic agents, diagnosis and treatment of this infection have been reviewed.

Results: Laboratory methods such as investigation of smear by potassium hydroxide and staining and cultural and molecular methods can be helpful in the diagnosis. Treatment of this infection with improved outcome is challenging. Medical therapy (topical and systemic treatments) is the first choice of treatment, but early surgical intervention and removal of the infected tissues are critical for effective cure. Lack of response to these therapies requires corneal transplantation. Unfortunately, loss of useful vision rate was found in patients even with treatment by antifungal agents.

Conclusions: The signs and symptoms of fungal keratitis are similar to those of other microbial keratitis. Use of diagnostic methods and early treatment are helpful for recovery in infected pediatric patients.
\end{abstract}

Keywords: Fungi, Cornea, Keratitis, Pediatric Patients, Aspergillus, Fusarium

\section{Context}

In pediatric patients (aged $\leq 16$ years), a proportion of microbial corneal diseases (non-viral) includes fungal keratitis (FK).The incidence rate of FK in microbial keratitis in pediatric patients in the United States, China, and South India were reported to be $18 \%, 48.7 \%$, and $54.2 \%$, respectively (1-3).

This infection is the most common cause of blindness in the world, major ophthalmologic problems, and serious ocular infections, particularly in hot and humid areas and in developing countries (4). The infection is mostly seen in immunocompromised patients (transplant recipients, diabetics, cancer, and HIV/AIDS sufferers), and in immunocompetent patients (5). Complications in some patients include corneal graft rejection, progressive cataracts, recurrent fungal infection, and secondary glaucoma due to use of topical steroids $(6,7)$. Only a few studies have been published on the clinical features of FK in pediatric patients. This review discusses the diagnosis and treatment of FK in pediatric patients for the best management of the infection.

\section{Evidence Acquisition}

In this narrative review, the most recently published data on pediatric fungal keratitis, respective prominent signs and symptoms, etiologic agents, and diagnosis and treatment of this infection have been reviewed.

\section{Results}

\subsection{Predisposing Factors}

This infection can present as an exogenous infection following the ingestion of foreign material, prior intraocular surgery, contact lens wear, and trauma, and also FK is associated with contaminated topical triamcinolone drop/ointment and brilliant blue $G$, as reported $(8,9)$. Trauma with vegetative materials, plants, metal, plastic pieces, firecrackers, pencils, and contact lenses are the most background for the ingestion of the fungal conidia directly in the corneal stroma $(10,11)$. According to Aruljyothi et al. 125 from 240 (53.4\%) patients had a history of trauma. Trauma with vegetative matter and plant were the most common risk factor in pediatric patients aged 1 to 7 years (38.6\%) and over 7 years (24.2\%), while in pediatric patients under 1 year old injury with dust particle (13.3\%) was common (3). In another study, contact lens 
wear was the most well known risk factor (33 cases, 40.7\%), followed by trauma (17 cases, $21.0 \%$ ) in children suffering from microbial keratitis (12). Symptoms of exogenous FK present within 48 hours of foreign body ingestion, but they present after 7 to 20 days in posttraumatic fungal endophthalmitis and postoperative cases (12).

Endogenous FK represents intraocular dissemination of an invasive fungal infection by Candida or Aspergillus species in systemic antibiotic or corticosteroid therapy. Malignancy, multiple congenital abnormalities, malnutrition, diabetes mellitus, intravenous drug use, and recent indwelling central venous catheter are the other endogenous intraocular dissemination $(2,13)$. In documented candidemia, within the first week following diagnosis, dilated retinal examination should be done preferably by an ophthalmologist (14). Systemic infections and malignancies are the main predisposing factors for FK in children younger than 4 years $(1,15)$. Systemic illness and previous ocular surgery in Taiwanese pediatric patients were reported to be $11.1 \%$ and $6.2 \%$, respectively (16).

\subsection{Mechanism of Infection}

Defect in the epithelial barrier by trauma and other risk factors can lead the fungi to the corneal stroma, where they multiply. Host inflammatory reaction and tissue necrosis may also occur. Fungi gain access to the anterior chamber and spread from the cornea into the sclera. At this time, the treatment and eradication of the organisms become extremely difficult (17). Coexisting ocular diseases caused by more than 1 microorganism (bacterial or viral with fungal) have been reported in the literature $(3,18-20)$.

\subsection{Signs and Symptoms}

Clinical features of FK are the same as those of other microbial infections (Figure 1) and must be differentiated from bacterial, herpetic, and acanthamoeba keratitis (21). Only serrated margins and raised slough were independently associated with FK (22) and exudates presented more frequently in cases suffering from filamentous FK, hypopyon, and fibrinous than in bacterial keratitis (22). Other signs are redness of the eye, blepharitis lid edema, satellite lesions, increasing eye pain, or discomfort or foreign body sensation, increased light sensitivity, raised slough, dry eyes, abnormalities and conjunctival congestion, corneal degeneration, and defective vision (4). In children, corneal ulcers, as defined by the corneal infiltrate, hypopyon, and perforations were reported with no significant difference between age groups (16). If 1 or 3 clinical features (raised slough, dry eyes, abnormalities, conjunctival congestion, corneal degeneration and defective vision pain, serrated margins, raised slough, hypopyon, redness of the eye, blepharitis lid edema, and fibrinous exudates) were present, the probabilities of FK would have been $63 \%$ and $83 \%$, respectively (22).The most infected site is cornea (23). Infection was reported the most with male preponderance, with a male-to-female ratio of $147 / 234,62.8 \%$ (3), but there was a report indicating no significant difference in sex (16). Infection frequently occurs in males in agricultural activities, especially during summer, and if children do not use any protection for the eyes, they become infected by the plant debris, leaf spots, vegetative garbage, seedling blight, and seed germination.

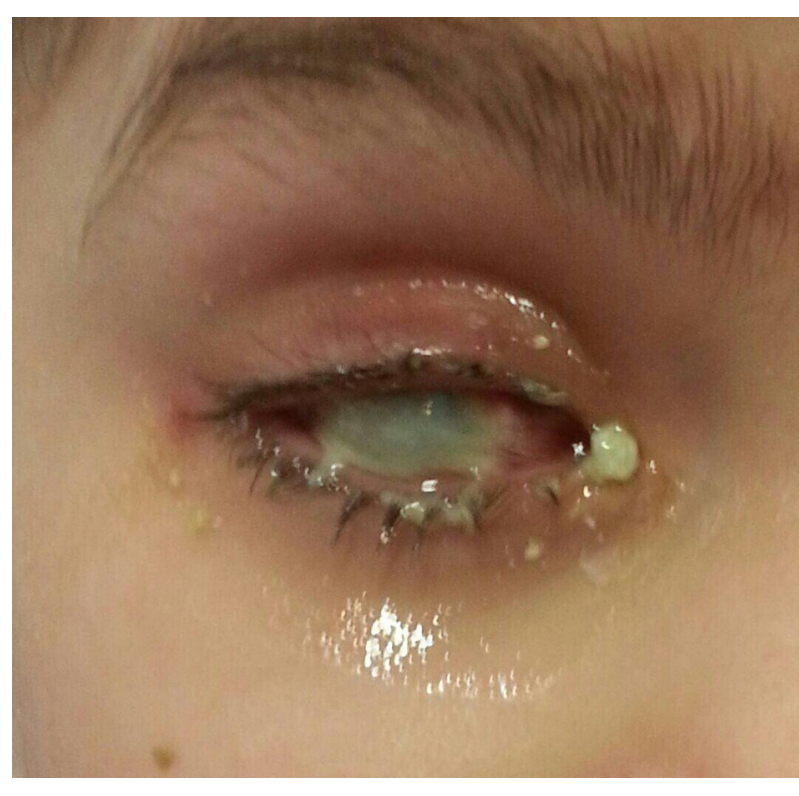

Figure 1. Mucoral Keratitis in 8 Years Old Immunocompotent Boy

Age distributions in microbial infected children were reported $45 / 78$ eyes in group with age $\leq 12$ years and 36/78 eyes in age $>12$ years (16). In Indian pediatric patients, infection was seen in age $\leq 1$ years in $15 / 234$ (6.4\%), 1 to 7 years $70 / 234$ (29.9\%), and > 7 years $149 / 234$ (63.7\%) (3). There was a higher incidence of FK during the monsoon than winter (47\% vs. 24\%) (24). There are different ratios in incidence of positive cultures during the first (January to June) and second (July to December) half of the year, ranging between 1 and $2.1(25)$.

\subsection{Etiologic Agents}

Fungal infections of the cornea are frequently caused by both filamentous and yeast species including Fusarium, Aspergillus, Rhizopus, Mucor, Paecilomyces, Scedosporium, Candida species, Phaeohyphomycetes, and Curvularia (26). Filamentous fungi now account for most of exogenous FK 
cases in tropical regions, whereas yeasts are the predominant endogenous pathogen in temperate climates. From filamentous fungi Fusarium and Aspergillus species and from yeast, Candida species are the main types of common pathogens, especially in tropical climates $(17,27)$. Fusarium was the most common (59.7\%) fungal species isolated from Indian children in a study in 2015 and in another study (1975) Aspergillus followed by Fusarium species were the major causative fungi (28). Common species of Fusarium were Fusarium solani, Fusarium moniliforme, and Fusarium oxysporum (29). The genus Aspergillus is the most common group of fungi in the environment and manifests in an invasive, colonizing, or allergic manner. Aspergillus endophthalmitis has been linked to endogenous etiologies in the disseminated form among the immunocompromised patients. The main species isolated from FK were reported to be Aspergillus flavus and Aspergillus fumigates (30). Other fungal species such as Mucorals (5) and Paecilomyces (31) were responsible for more limited cases.

Yeast fungi (e.g., genus Candida) are responsible for some cases of FK in the world. The reported range of Candida keratitis is between $9.3 \%$ to $14 \%(6,32)$. This organism causes endogenous ocular infections with chronic erosions/ulceration in immunocompromised hosts and compromised corneal surface (17) after trauma. From phaeohyphomycosis family, Curvularia is the third most prevalent filamentous hyphomycetes in corneal isolates in adults, with rate of $12 \%$ (32-34). Unfortunately, there is no report on FK by these fungi in pediatric patients.

\subsection{Diagnosis}

Early diagnosis and antifungal therapy are necessary for the best management of FK and can prevent further complications such as loss of vision, endophthalmitis, blindness, and amblyopia (35). Use of Slit lamp biomicroscope and confocal microscopy can help careful examination and evaluation of the site of the infection, any perforation, ulcer size and depth, infiltration, and abscess formation $(36,37)$. These microscopes can serve as rapid and sensitive diagnostic tools and follow- up of the patients. Intraocular samples from vitreous (or aqueous) or the removed cornea may be necessary for the diagnosis of patients. Corneal scraping in pediatric patients, especially those $<2$ years needs sedation or general anesthesia (38). Sampling is not necessary in patients with documented candidemia or fungemia, when infection has extended into the aqueous and in patients with funduscopic findings, typical for Candida and Aspergillus chorioretinitis.

\subsubsection{Direct Study}

The first step of quick lab diagnosis for confirmation is scraping or biopsy of infected lesion and investigating the direct microscopic smear detection by potassium hydroxide (KOH) or staining (8). Corneal biopsy may be required if smears and cultures of scraping materials are negative and clinical improvement is absent after 3 to 4 days. Sensitivity of staining varies in the studies. Potassium hydroxide wet mount examination is a conventional method for the diagnosis of FK (39). The sensitivity rates of this method were reported 76.3\% (37), 91.0\% (40), and 84.62\% (6). Calcofluor white (CFW) highlights the fungal cell wall and increases the sensitivity of $\mathrm{KOH}$ to $91.4 \%$ (33), 99.0\% (40), and 99.44\% (6). Gram stain for detecting fungi in corneal scrapings identifies yeast but cannot visualize the hyphae of molds, with the reported sensitivity rates ranging from $42.1 \%$ (41) to $88.2 \%(40)$. Other staining can be helpful to the diagnosis of fungal hyphae in scraping samples or tissue sections. The sensitivities of Methylene Blue, Periodic acid Schiff, and Geimsa were reported to be $92.31 \%$ (6), 90.7\%6 (6) and $85.1 \%(33,40)$, respectively. Gomori methenamine silver is a staining that identifies hyphal fragments; unfortunately, we did not find any study reporting the sensitivity of this method.

\subsubsection{Culture}

The resistance of pathogenic fungi to antifungal agents was reported $(42,43)$. The isolation of fungi from the eye specimens and determination of susceptibility pattern of the organisms are important for the best treatment of respective patients. Fungal culture is the cornerstone for the documented diagnosis of $\mathrm{FK}$, and thus recognition of pathogenic fungi is important for distribution, prevention, and treatment of FK (25). In patients with suspected endogenous endophthalmitis, blood culture can be useful for the diagnosis of etiologic agents (Figures 2,3).

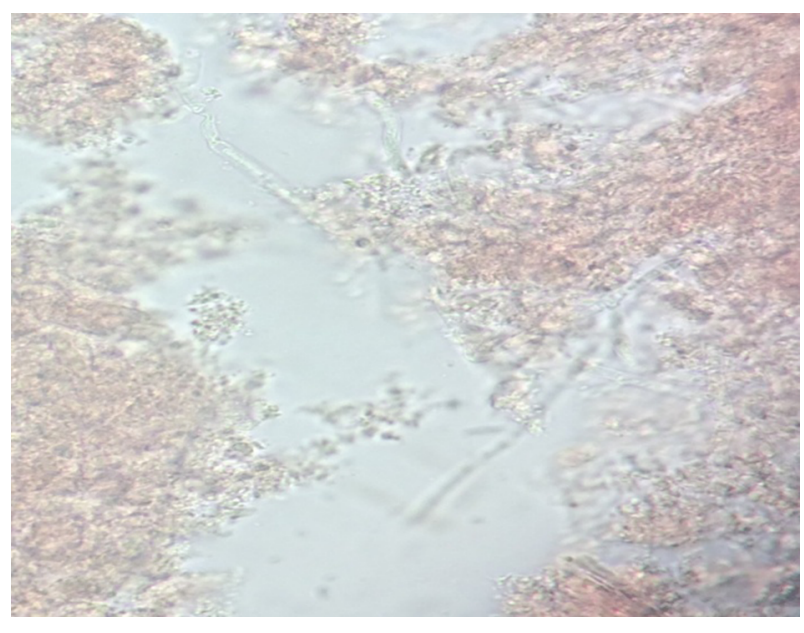

Figure 2. Hypgea of Filamentus Fungi in Direct Smear with Pottasium Hydroxide 


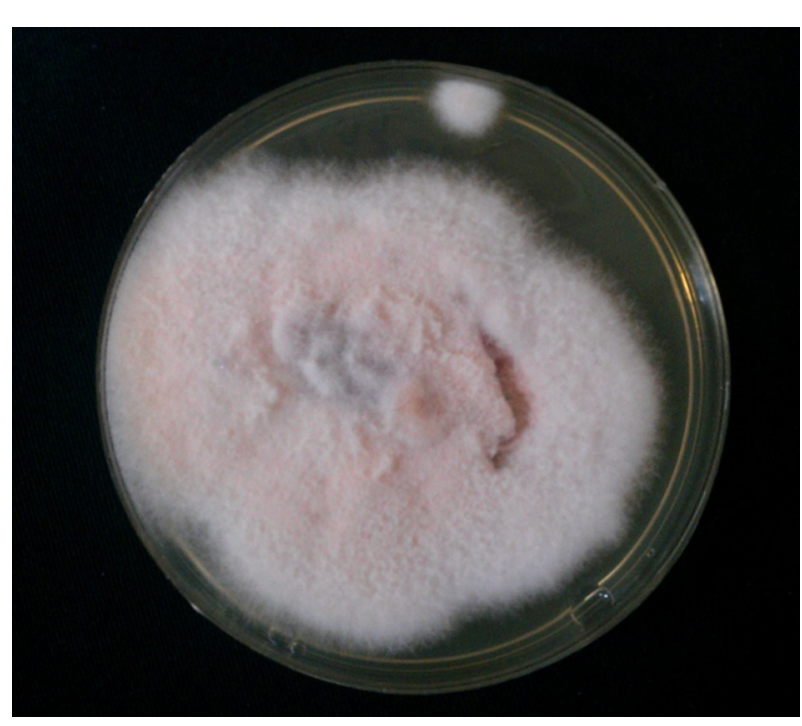

Figure 3. Fusarium Species Isolated from Patient with Fusarium Keratitis

The rates of positive fungal culture were reported to be 29.7\% (25), 68.4\% (41), 81.5\% (29), and 89.8\% (6). Sabouraud dextrose agar is a particular culture medium, and fungi typically grow best on it. Diagnosis of isolates is based on microscopic examination and morphologic characteristics of isolates in the culture media. Isolated fungi are identified by lactophenol cotton blue wet mount smear and chemical laboratory tests such as API 20 C AUX (application programming interface). Sensitivity patterns of the isolates can be determined by micro dilution method and E test $(42,43)$. Since fungi (Aspergillus, Candida, Rhizopus, and Scopulariopsis species) are recognized in 3 to $28 \%$ of healthy conjunctival sacs (17), interpretation of the isolates must be performed along with clinical signs and symptoms of the patients.

\subsubsection{Molecular Methods}

There are many reports on the molecular diagnosis of systemic fungal infections (source of endogenous FK) in blood (44-46) and FK in the eye samples of patients (41, 47). The sensitivity rates of molecular methods for the detection of FK were reported to be $81.6 \%$ (41), 92.6\% (47), and $100 \%$ for Aspergillus keratitis by nested PCR assay (30). There are some advantages and limitations for the diagnosis of FK by molecular methods. These methods are more sensitive, effective, and rapid for FK diagnose than stain and culture methods. The time taken for PCR assay is 4 to 8 hours (within a working day), whereas positive fungal cultures may take many days. Due to the lack of sophisticated facilities in routine laboratory procedures, it can serve only as a complementary assay and cannot replace conventional ones. Fungal PCR must be added as a screening diagnostic test, when an early differentiation is necessary between fungal and microbial types of keratitis.

\subsection{Treatment}

Based on clinical features, fungal eye infections have had poor outcomes and are difficult to diagnose and treat. Prognosis of FK depends on early diagnosis and treatment. Unfortunately, significant visual impairment has been observed even with early treatment by systemic/topical antifungal agents. Medical therapy (topical and systemic treatments) is the first choice of treatment, but it sometimes fails, and urgent surgical intervention is warranted (8). Lack of response to these therapies requires corneal transplantation (4).

Small ulcer and superficial infections respond well, but deep stromal infections, large ulcer size (>14 mm), presence of hypopyon concomitant sclera, or intraocular involvement are not responsive enough in topical therapy $(48,49)$. Most antifungals are fungi static; therefore, successful treatment needs to continue for prolonged periods (50), and initially be applied hourly with subsequent modification based on response. Bacterial coinfection can be protected by broad-spectrum antibiotics. There are no clinical trial studies about Candida keratitis, and evidence of the treatment of endogenous infection was reported in the form of case reports. The treatment of choice for Candida infection could be topical amphotericin $0.15 \%$ (0.1\% $0.3 \%$ ), topical capsofungin $0.5 \%$, econazole $1 \%$, and fluconazole $2 \%$, moreover, alternatives include natamycin $5 \%$ and clotrimazole $1 \%(8,32)$. Capsofungin is more expensive and its use is limited. Posaconazole concentrations in the vitreous are not high and not recommended for the treatment (51). In severe cases, to improve the quality and reduce treatment period, subconjunctival fluconazole and intravitreal or intra cameral amphotericin B injection seem to be effective (52). Voriconazole is available for intravenous or oral administration and is effective against $C$. glabrata and C. krusei, which are resistant to fluconazole (6). The concentration of this antifungal in the vitreous is reported to be approximately $40 \%$ of serum concentration (51). The concentrations of echinocandins in the vitreous cannot be evaluated (51) and its use in the treatment of Candida endophthalmitis is unclear.

Based on patient's condition, treatment of mold FK includes only topical antifungal agent or combined with removal of infected materials by vitrectomy. Systemic antifungal therapy is suggested in patients with severe fungal infections or in immunocompromised patients. Filamentous fungi were mostly sensitive to natamycin, amphotericin B and terbinafine (29). The first choice for treating hyphomycetic keratitis is topical natamycin $(5 \mathrm{mg} / \mathrm{mL})$. 
Coinfection due to natamycin contaminated with Pseudomonas aeruginosa was also reported $(19,53)$.

The role of topical voriconazole in the management of FK, especially in Aspergillus species and some uncommon fungal pathogens such as Paecilomyces lilacinus, was reported (54-56). Voriconazole is a good choice for the treatment of FK due to deep penetration in all tissues. Treatment with voriconazole in over 40 clinical cases with a broad range of fungal pathogens resistant to some antifungals like amphotericin B was reported (57). In studies comparing voriconazole and natamycin, no significant differences were found in scar size and perforations in patients treated with voriconazole and natamycin (58), but natamycin was found to be more effective in visual acuity and healing corneal ulcers (59).

Other antifungals in triazole generation including posaconazole and ravuconazole have been shown as effective antifungal drugs with few side effects (55). Voriconazole and other triazoles demonstrated a high capacity for the treatment of mold FK, however, itraconazole and caspofungin were not effective in the treatment of Fusarium species infections (49).

according to food and drug administration (FDA), natamycin, the polyene antifungal agent, is the first line therapy and can only be administered topically for the treatment of FK. There are reports about resistant natamycin Aspergillus species or natamycin unresponsive mycotic keratitis $(49,51)$. In the ophthalmic literature, the use of voriconazole and amphotericin B topical was reported for the treatment of filamentous FK $(51,55)$. Voriconazole had more permeability than natamycin in penetration in intact epithelium $(55,57)$. Natamycin suspension $(5 \mathrm{mg} / \mathrm{mL})$ and amphotericin B drop $(1.5 \mathrm{mg} / \mathrm{mL})$ have been used for the treatment of topical FK (60). Combination of systemic antifungal medications with topical treatment was suggested for immunocompromised patients with severe infections (60). Treatment with oral posaconazole has been reported to be successful in those patients not responsive to voriconazole therapy (61). As an early antifungal therapy is necessary for treatment, injection of amphotericin B or voriconazole is warranted for cases infected by mold endophthalmitis $(51,62)$. The priority treatment of FK with immunotherapies was considered in the literature (63). Monoclonal antibody to Poly$\mathrm{N}$-acetyl glucosamine demonstrated protection and treatment against many important fungi. The optimal duration of FK therapy depends on epithelial healing. Patients must be examined by an ophthalmologist continuously. However, this time is at least one to several months in exogenous infections and for endogenous endophthalmitis, the duration of therapy depends on the response to systemic fungal infections, which usually extends to several months.

Fungal keratitis more likely requires surgical intervention, compared to bacterial or Acanthamoeba keratitis (11). Penetrating and lamellar keratoplasty are effective treatments and provide useful vision for FK in patients not responsive to antifungal medications. Removal of the infected epithelium around the lesion may increase penetration of the antifungal agents. Early surgical intervention and removal of the infected tissues are critical for effective cure (6). Surgical intervention in the acute phase was necessary in $23 \%$ of the patients (32); this rate was higher (67\%) in patients with a history of corneal transplant (64). According to Aruljyothi et al. 5 out of 63 patients (7.9\%) with prior empiric treatment and 12 out of 171 patients (7\%) without such treatment underwent therapeutic keratoplasty (3).

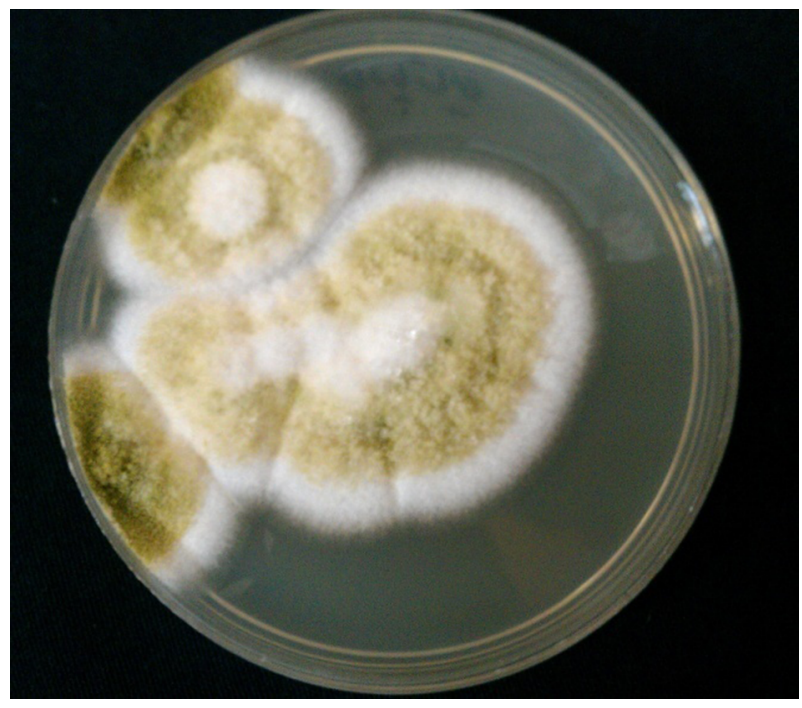

Figure 4. Aspergillus Isolated from Aspergillus Keratitis

\section{Conclusions}

Pediatric microbial keratitis is an uncommon, but potentially serious condition. Rapid diagnosis of the infection may be possible by mycological investigations or scoring clinical features. The use of laboratory methods based on direct smear by potassium hydroxide and staining can be reliable and helpful for early diagnosis and initiation of antifungal therapy. Also, cultural and molecular methods can be helpful in diagnosis of etiologic agents. The search for early and efficient diagnosis of this infection needs to be continued. Use of proper and novel antifungal agents can improve the outcome of the disease. Thus, continued treatment for several months until the infiltrate is 
resolved and epithelial stroma healed is of paramount inmportance.

\section{Acknowledgments}

We are grateful to Hassan Khajehei for his help with language editing.

\section{Footnotes}

Authors' Contribution: Parisa Badiee designed the study and drafted the article, Mahmood Nejabat analyzed and interpreted the data and critically revised for important intellectual content, Maral Choopanizadeh searched in the literature, acquired data, and drafted the article. All the authors have approved the final version of the manuscript and agreed to be responsible for all aspects of the work in ensuring that questions related to the accuracy or integrity of any part of the work are appropriately investigated and resolved.

Financial Disclosure: The authors do not have any financial or other relationships that could be regarded as a conflict of interest.

\section{References}

1. Cruz OA, Sabir SM, Capo H, Alfonso EC. Microbial keratitis in childhood. Ophthalmology. 1993;100(2):192-6. doi: 10.1016/S01616420(93)31671-4. [PubMed: 8437826].

2. Song $\mathrm{X}, \mathrm{Xu}$ L, Sun S, Zhao J, Xie L. Pediatric microbial keratitis: a tertiary hospital study. Eur J Ophthalmol. 2012;22(2):136-41. doi: 10.5301/EJO.2011.8338. [PubMed: 21574163].

3. Aruljyothi L, Radhakrishnan N, Prajna VN, Lalitha P. Clinical and microbiological study of paediatric infectious keratitis in South India: a 3-year study (2011-2013). Br J Ophthalmol. 2016;100(12):1719-23. doi: 10.1136/bjophthalmol-2015-307631. [PubMed: 26917675].

4. Badiee P. Mycotic keratitis, a state-of-the-art review. Jundishapur J Microbiol. 2013;6(5) doi: 10.5812/jjm.8561.

5. Badiee P, Jafarpour Z, Alborzi A, Haddadi P, Rasuli M, Kalani M. Orbital mucormycosis in an immunocompetent individual. Iran J Microbiol. 2012;4(4):210-4. [PubMed: 23205254].

6. Xie L, Dong X, Shi W. Treatment of fungal keratitis by penetrating keratoplasty. $B r$ J Ophthalmol. 2001;85(9):1070-4. doi: 10.1136/bjo.85.9.1070. [PubMed: 11520759].

7. Jain V, Maiti A, Shome D, Borse N, Natarajan S. Aspergillusinduced malignant glaucoma. Cornea. 2007;26(6):762-3. doi: 10.1097/ICO.0b013e31805fc37b. [PubMed: 17592335].

8. Thomas PA. Fungal infections of the cornea. Eye (Lond). 2003;17(8):852-62. doi:10.1038/sj.eye.6700557. [PubMed: 14631389].

9. Mikosz CA, Smith RM, Kim M, Tyson C, Lee EH, Adams E, et al. Fungal endophthalmitis associated with compounded products. Emerg Infect Dis. 2014;20(2):248-56. doi: 10.3201/eid2002.131257. [PubMed: 24447640].

10. Singh G, Palanisamy M, Madhavan B, Rajaraman R, Narendran K, Kour A, et al. Multivariate analysis of childhood microbial keratitis in South India. Ann Acad Med Singapore. 2006;35(3):185-9. [PubMed: 16625268].

11. Gopinathan U, Sharma S, Garg P, Rao GN. Review of epidemiological features, microbiological diagnosis and treatment outcome of microbial keratitis: experience of over a decade. Indian J Ophthalmol. 2009;57(4):273-9. doi: 10.4103/0301-4738.53051. [PubMed: 19574694].
12. Chakrabarti A, Shivaprakash MR, Singh R, Tarai B, George VK, Fomda BA, et al. Fungal endophthalmitis: fourteen years' experience from a center in India. Retina. 2008;28(10):1400-7. doi 10.1097/IAE.0b013e318185e943. [PubMed: 19009680].

13. Sridhar J, Flynn HJ, Kuriyan AE, Miller D, Albini T. Endogenous fungal endophthalmitis: risk factors, clinical features, and treatment outcomes in mold and yeast infections. J Ophthalmic Inflamm Infect. 2013;3(1):60. doi: 10.1186/1869-5760-3-60. [PubMed: 24053550].

14. Pappas PG, Kauffman CA, Andes D, Benjamin DJ, Calandra TF, Edwards JJ, et al. Clinical practice guidelines for the management of candidiasis: 2009 update by the Infectious Diseases Society of America. Clin Infect Dis. 2009;48(5):503-35. doi:10.1086/596757. [PubMed: 19191635].

15. Clinch TE, Palmon FE, Robinson MJ, Cohen EJ, Barron BA, Laibson PR Microbial keratitis in children. Am J Ophthalmol. 1994;117(1):65-71. doi: 10.1016/S0002-9394(14)73016-8. [PubMed: 8291594].

16. Hsiao CH, Yeung L, Ma DH, Chen YF, Lin HC, Tan HY, et al. Pediatric microbial keratitis in Taiwanese children: a review of hospital cases. Arch Ophthalmol. 2007;125(5):603-9. doi: 10.1001/archopht.125.5.603. [PubMed: 17502497].

17. Elshiek H, Pineda R. Fungal keratitis. Al-Basar Int J Ophthalmol. 2015;3(1):3-5. doi: 10.4103/1858-6538.169310.

18. Manikandan P, Varga J, Kocsube S, Anita R, Revathi R, Nemeth TM, et al. Epidemiology of Aspergillus keratitis at a tertiary care eye hospital in South India and antifungal susceptibilities of the causative agents. Mycoses. 2013;56(1):26-33. doi: 10.1111/j.1439-0507.2012.02194.x. [PubMed: 22487304].

19. Krishnan T, Sengupta S, Reddy PR, Ravindran RD. Secondary pseudomonas infection of fungal keratitis following use of contaminated natamycin eye drops: a case series. Eye (Lond). 2009;23(2):477-9. doi: 10.1038/eye.2008.290. [PubMed: 18820656].

20. Lin TT, Wei RH, Yang RB, Huang Y, Zhang C, Ning YX, et al. Fungal Keratitis Associated with Viral Keratitis. Chin Med J (Engl). 2015;128(20):2823-5. doi: 10.4103/0366-6999.167367. [PubMed: 26481755].

21. Dalmon C, Porco TC, Lietman TM, Prajna NV, Prajna L, Das MR, et al The clinical differentiation of bacterial and fungal keratitis: a photographic survey. Invest Ophthalmol Vis Sci. 2012;53(4):1787-91. doi: 10.1167/iovs.11-8478. [PubMed: 22395880].

22. Thomas PA, Leck AK, Myatt M. Characteristic clinical features as an aid to the diagnosis of suppurative keratitis caused by filamentous fungi. Br J Ophthalmol. 2005;89(12):1554-8. doi: 10.1136/bjo.2005.076315. [PubMed: 16299128].

23. Al-Otaibi AG. Non-viral microbial keratitis in children. Saudi J Ophthalmol. 2012;26(2):191-7. doi: 10.1016/j.sjopt.2011.10.002. [PubMed: 23960991].

24. Lam DS, Houang E, Fan DS, Lyon D, Seal D, Wong E, et al. Incidence and risk factors for microbial keratitis in Hong Kong: comparison with Europe and North America. Eye (Lond). 2002;16(5):608-18. doi: 10.1038/sj.eye.6700151. [PubMed: 12194077].

25. Sun XG, Zhang Y, Li R, Wang ZQ, Luo SY, Jin XY, et al. Etiological analysis on ocular fungal infection in the period of 1989 - 2000. Chin Med J (Engl). 2004;117(4):598-600. [PubMed: 15109456].

26. Lakhundi S, Siddiqui R, Khan NA. Pathogenesis of microbial keratitis. Microb Pathog. 2017;104:97-109. doi: 10.1016/j.micpath.2016.12.013. [PubMed: 27998732].

27. Bennett JE, Dolin R, Blaser MJ. Principles and practice of infectious diseases. Elsevier Health Sciences; 2014.

28. Perkins RE, Kundsin RB, Pratt MV, Abrahamsen I, Leibowitz HM Bacteriology of normal and infected conjunctiva. J Clin Microbiol. 1975;1(2):147-9. [PubMed: 1176598].

29. Xie L, Zhai H, Zhao J, Sun S, Shi W, Dong X. Antifungal susceptibility for common pathogens of fungal keratitis in Shandong Province, China. Am J Ophthalmol. 2008;146(2):260-5. doi: 10.1016/j.ajo.2008.04.019. [PubMed: 18547535]. 
30. Badiee P, Alborzi A, Nejabat M. Detection of Aspergillus keratitis in ocular infections by culture and molecular method. Int Ophthalmol. 2011;31(4):291-6. doi: 10.1007/s10792-011-9457-5. [PubMed: 21750945]

31. Toker E, Ziyade N, Atici S, Eda KK, Turel O, Toprak D, et al. Postoperative keratitis due to Paecilomyces: a rare pediatric case. Pan Afr Med J. 2016;24:317. doi: 10.11604/pamj.2016.24.317.9772. [PubMed: 28154672].

32. Iyer SA, Tuli SS, Wagoner RC. Fungal keratitis: emerging trends and treatment outcomes. Eye Contact Lens. 2006;32(6):267-71. doi: 10.1097/01.icl.0000249595.27520.2e. [PubMed: 17099386].

33. Gopinathan U, Garg P, Fernandes M, Sharma S, Athmanathan S, Rao GN. The epidemiological features and laboratory results of fungal keratitis: a 10-year review at a referral eye care center in South India. Cornea. 2002;21(6):555-9. [PubMed:12131029].

34. Kermani NK, Aggarwal SP. Isolated post-operative Aspergillus niger endophthalmitis. Eye (Lond). 2000;14 ( Pt 1):114-6. doi: 10.1038/eye.2000.32. [PubMed: 10755121].

35. Kunimoto DY, Sharma S, Reddy MK, Gopinathan U, Jyothi J, Miller D, et al. Microbial keratitis in children. Ophthalmology. 1998;105(2):2527. doi: 10.1016/S0161-6420(98)92899-8. [PubMed: 9479283].

36. Avunduk AM, Beuerman RW, Varnell ED, Kaufman HE. Confocal microscopy of Aspergillus fumigatus keratitis. Br J Ophthalmol. 2003;87(4):409-10. doi:10.1136/bjo.87.4.409. [PubMed: 12642300].

37. Brasnu E, Bourcier T, Dupas B, Degorge S, Rodallec T, Laroche L, et al. In vivo confocal microscopy in fungal keratitis. Br J Ophthalmol. 2007;91(5):588-91. doi: 10.1136/bjo.2006.107243. [PubMed: 17151059].

38. Vajpayee RB, Ray M, Panda A, Sharma N, Taylor HR, Murthy GV, et al. Risk factors for pediatric presumed microbial keratitis: a case-control study. Cornea. 1999;18(5):565-9. doi: 10.1097/00003226-19990900000009. [PubMed:10487431].

39. Jain AK, Bansal R, Felcida V, Rajwanshi A. Evaluation of impression smear in the diagnosis of fungal keratitis. Indian J Ophthalmol. 2007;55(1):33-6. doi: 10.4103/0301-4738.29492. [PubMed: 17189884].

40. Sharma S, Kunimoto DY, Gopinathan U, Athmanathan S, Garg P, Rao GN. Evaluation of corneal scraping smear examination methods in the diagnosis of bacterial and fungal keratitis: a survey of eight years of laboratory experience. Cornea. 2002;21(7):643-7. doi: 10.1097/00003226-200210000-00002. [PubMed: 12352078].

41. Badiee P, Nejabat M, Alborzi A, Keshavarz F, Shakiba E. Comparative study of Gram stain, potassium hydroxide smear, culture and nested PCR in the diagnosis of fungal keratitis. Ophthalmic Res 2010;44(4):251-6. doi: 10.1159/000313988. [PubMed: 20699629].

42. Badiee P, Alborzi A, Moeini M, Haddadi P, Farshad S, Japoni A, et al. Antifungal susceptibility of the aspergillus species by Etest and CLSI reference methods. Arch Iran Med. 2012;15(7):429-32. [PubMed 22724880].

43. Badiee P, Alborzi A, Shakiba E, Ziyaeyan M, Rasuli M. Molecular Identification and In-Vitro Susceptibility of Candida albicans and C. dubliniensis Isolated from Immu-nocompromised Patients. Iran Red Crescent Med J. 2009;11(4):391-7.

44. Badiee P, Alborzi A. Assessment of a real-time PCR method to detect human non-cryptococcal fungal meningitis. Arch Iran Med. 2011;14(6):381-4. [PubMed: 22039841].

45. Mirhendi H, Moazeni M, Nikaeen M, Makimura K. Typing of Aspergillus fumigatus and Aspergillus niger strains by random amplification of polymorphic DNA analysis using a six primer set. Shiraz E Med J. 2009;10(4):190-200.

46. Badiee P, Kordbacheh P, Alborzi A, Ramzi M, Shakiba E. Molecular detection of invasive aspergillosis in hematologic malignancies. In fection. 2008;36(6):580-4. doi: 10.1007/s15010-008-7385-8. [PubMed: 18854937].

47. Ferrer C, Alio JL. Evaluation of molecular diagnosis in fungal keratitis. Ten years of experience.JOphthalmic Inflamm Infect. 2011;1(1):15-22. doi: 10.1007/s12348-011-0019-9. [PubMed: 21475656].

48. Nielsen SE, Nielsen E, Julian HO, Lindegaard J, Hojgaard K, Ivarsen A, et al. Incidence and clinical characteristics of fungal keratitis in a Dan- ish population from 2000 to 2013. Acta Ophthalmol. 2015;93(1):54-8. doi: 10.1111/aos.12440. [PubMed: 24836583].

49. Lalitha P, Shapiro BL, Srinivasan M, Prajna NV, Acharya NR, Fothergill AW, et al. Antimicrobial susceptibility of Fusarium, Aspergillus, and other filamentous fungi isolated from keratitis. Arch Ophthalmol. 2007;125(6):789-93. doi: 10.1001/archopht.125.6.789. [PubMed: 17562990].

50. Mravii I, Dekaris I, Gabri N, Romac I, Glavota V, Mlinari E. An overview of fungal keratitis and case report on trichophyton keratitis. In Tech Open. 2012 doi: 10.5772/33695.

51. Riddell J, Comer GM, Kauffman CA. Treatment of endogenous fungal endophthalmitis: focus on new antifungal agents. Clin Infect Dis. 2011;52(5):648-53. doi: 10.1093/cid/ciq204. [PubMed: 21239843].

52. Yoon KC, Jeong IY, Im SK, Chae HJ, Yang SY. Therapeutic effect of intracameral amphotericin $\mathrm{B}$ injection in the treatment of fungal keratitis. Cornea. 2007;26(7):814-8. doi: 10.1097/ICO.0b013e31806c791e. [PubMed: 17667615].

53. Alfonso EC, Cantu-Dibildox J, Munir WM, Miller D, O’Brien TP, Karp $\mathrm{CL}$, et al. Insurgence of Fusarium keratitis associated with contact lens wear. Arch Ophthalmol. 2006;124(7):941-7. doi: 10.1001/archopht.124.7.ecs60039. [PubMed:16769827].

54. Ansari Z, Miller D, Galor A. Current Thoughts in Fungal Keratitis: Diagnosis and Treatment. Curr Fungal Infect Rep. 2013;7(3):20918. doi: 10.1007/s12281-013-0150-110.1007/s12281-013-0150-1. [PubMed: 24040467].

55. Hariprasad SM, Mieler WF, Lin TK, Sponsel WE, Graybill JR. Voriconazole in the treatment of fungal eye infections: a review of current literature. BrJOphthalmol. 2008;92(7):871-8. doi:10.1136/bjo.2007.136515. [PubMed: 18577634].

56. Prajna NV, Mascarenhas J, Krishnan T, Reddy PR, Prajna L, Srinivasan $\mathrm{M}$, et al. Comparison of natamycin and voriconazole for the treatment of fungal keratitis. Arch Ophthalmol. 2010;128(6):672-8. doi: 10.1001/archophthalmol.2010.102. [PubMed: 20547942].

57. Sharma S, Das S, Virdi A, Fernandes M, Sahu SK, Kumar Koday N, et al. Re-appraisal of topical $1 \%$ voriconazole and $5 \%$ natamycin in the treatment of fungal keratitis in a randomised trial. Br J Ophthalmol. 2015;99(9):1190-5. doi: 10.1136/bjophthalmol-2014-306485. [PubMed: 25740805].

58. Lalitha P, Sun CQ, Prajna NV, Karpagam R, Geetha M, O'Brien KS, et al. In vitro susceptibility of filamentous fungal isolates from a corneal ulcer clinical trial. Am J Ophthalmol. 2014;157(2):318-26. doi: 10.1016/j.ajo.2013.10.004. [PubMed: 24439440].

59. Riddell Iv J, McNeil SA, Johnson TM, Bradley SF, Kazanjian PH, Kauffman CA. Endogenous Aspergillus endophthalmitis: report of 3 cases and review of the literature. Medicine (Baltimore). 2002;81(4):311-20. doi: 10.1097/00005792-200207000-00007. [PubMed: 12169886].

60. Thomas PA, Kaliamurthy J. Mycotic keratitis: epidemiology, diagnosis and management. Clin Microbiol Infect. 2013;19(3):210-20. doi: 10.1111/1469-0691.12126. [PubMed: 23398543].

61. Tu EY, McCartney DL, Beatty RF, Springer KL, Levy J, Edward D. Successful treatment of resistant ocular fusariosis with posaconazole (SCH-56592). Am J Ophthalmol. 2007;143(2):222-7. doi:10.1016/j.ajo.2006.10.048. [PubMed: 17258521].

62. Kernt M, Neubauer AS, De Kaspar HM, Kampik A. Intravitreal voriconazole: in vitro safety-profile for fungal endophthalmitis. Retina. 2009;29(3):362-70. doi: 10.1097/IAE.0b013e31818d4b9b. [PubMed: 18997637].

63. Zhao G, Zaidi TS, Bozkurt-Guzel C, Zaidi TH, Lederer JA, Priebe GP, et al. Efficacy of Antibody to PNAG Against Keratitis Caused by Fungal Pathogens. Invest Ophthalmol Vis Sci. 2016;57(15):6797-804. doi: 10.1167/iovs.16-20358. [PubMed: 28002842].

64. Jurkunas U, Behlau I, Colby K. Fungal keratitis: changing pathogens and risk factors. Cornea. 2009;28(6):638-43. doi: 10.1097/ICO.ob013e318191695b. [PubMed: 19512908]. 